\title{
Hesperidin and naringin, two main flavonoids of citrus fruit, modulate bone metabolism in gonad-intact senescent male rats
}

\author{
V. Habauzit ${ }^{1}$, A. Trzeciakiewicz ${ }^{1}$, J. Mardon $^{1}$, A. Gil-Izquierdo ${ }^{2}$, C. Morand ${ }^{1}$, M. J. Davicco ${ }^{1}$, \\ P. Lebecque ${ }^{1}$, V. Coxam ${ }^{1}$ and M. N. Horcajada ${ }^{1}$ \\ ${ }^{1}$ INRA de Clermont-Theix, UMR 1019, Unité de Nutrition Humaine, Equipe Alimentation, Squelette et Métabolismes, \\ Saint-Genès Champanelle, France and ${ }^{2}$ CEBAS-CSIC, Departamento de Ciencia y Tecnología de Alimentos, \\ Espinardo - Murcia, Spain
}

Among fruit, citrus varieties are important sources of polyphenolic compounds, which could be responsible for the health-promoting effects. In the present study the effect of hesperidin (Hp) and naringin (Nar), the two major citrus flavonoids (flavanones), in regulating bone metabolism was examined in elderly male rats.

Forty-eight 20-month-old gonad-intact male Wistar rats were randomized into four groups. The control group received a casein-based diet, while the other three groups were fed for $90 \mathrm{~d}$ the same diet but supplemented with $(\mathrm{g} / \mathrm{kg}) 5 \mathrm{Hp}, 5 \mathrm{Nar}$ or a mix of $\mathrm{Hp}+\mathrm{Nar}$ (2.5 each). Femoral mineral density and strength and bone turnover markers (urine deoxypyridinoline and osteocalcin) were measured. Plasma and serum levels of leptin, total cholesterol, TAG, insulin, glucose, insulin-like growth factor-1, IL-6, TNF $\alpha$, ferric-reducing ability power, $\mathrm{NO}$ and circulating flavanones were also determined.

During the experiment body weights for each group were stable and were unaffected by flavanone intake. However, an effect of Hp and $\mathrm{Hp}+\mathrm{Nar}$ on body composition was suggested by a decrease in plasma leptin. Post-mortem examination showed that daily Hp intake improved bone mineral density compared with the control group, for all bone sites examined. While the same pattern was observed for the animals fed the mix of flavanones, only metaphyseal density was affected by Nar consumption. Nevertheless, femoral strength was not affected by flavanones. Bone resorption was slowed down by $\mathrm{Hp}$ and Nar whereas accretion remained unchanged. Flavanones improved lipid profiles but no changes in plasma insulin and glucose were detected. While TNF $\alpha$ was undetectable in serum for all groups, the consumption of flavanones appeared to prevent the increase in serum IL-6 associated with aging-related inflammation. In these conditions a slight decrease in NO production was observed for animals consuming flavanones, suggesting modulation of the oxidative status. Finally, circulating flavanones were only detected in the plasma of rats receiving Hp and/or Nar.

These results suggest that both $\mathrm{Hp}$ and Nar affect bone metabolism in gonad-intact senescent male rats. Moreover, blood lipid profiles are clearly improved by flavanone intake. Potential anti-inflammatory and antioxidant effects of these compounds remain to be confirmed. 